tiny minority, ideally, one should discuss with the radiologist beforehand which radiological techniques would be most likely to be helpful.

ERNEST P WORRALI

Division of Psychiatry,

Southern General Hospital,
Glasgow G51 4TF

Vecchio TJ. Predictive value of a single diagnostic test in unselected
$1966 ; 274: 1171-3$.

\section{Mean platelet volume in myocardial infarction}

SIR,-Dr J van der Lelie and Dr J A C Brakenhoff conclude their letter (12 November, p 1471): "measurement of the mean platelet volume in the coronary care unit has no clinical relevance." This negative assertion must be in serious doubt as it is based on a study of 12 patients with myocardial infarction, whereas the study by $\mathrm{Dr} \mathrm{H} \mathrm{A}$ Cameron and others (13 August, p 449) came to the opposite conclusion after studying mean platelet volume in 100 men with myocardial infarction using the same methodology (Coulter counter S plus). We should like to make some observations about methodology, theory, and interpretation.

As platelets are heterogenous for size and density it is important to measure a population truly representative of those circulating in whole blood. Recovery in our study (13 August, p 456) was 93 (SD 5)\% of those platelets in whole blood. If the recovery of measured platelets fell below $85 \%$ no difference in mean platelet volume was seen compared with controls, as the larger platelets were then lost in the velocity sedimentation used. Methodological problems also arise with the Coulter $S$ plus as it sizes platelets in the presence of red cells. The fact that the results of Dr Cameron and others were in accordance with ours, however, both at the time of infarct and 6-7 weeks later strengthens both papers. Dr van der Lelie and Dr Brakenhoff used edetic acid as anticoagulant, which causes swelling of platelets. ${ }^{1}$ Their mean platelet volume in myocardial infarct of $6.5 \mathrm{fl}$ is therefore surprising as it is smaller than $\mathrm{Dr}$ Cameron's using edetic acid $(9.07 \mathrm{fl})$ or ours $(7 \cdot 3 \mathrm{fl})$ using sodium citrate and prostaglandin $E_{1}$, which does not cause platelet swelling (and inhibits shape change). ${ }^{2}$ Furthermore, we have confirmed our results in a further study of 200 patients.

Their statement: "Mean platelet volume has been shown to be inversely and non-linearly related to platelet count, both in normal. controls and in a hospital population" is a gross oversimplification of a complex relation. Before the link between mean platelet volume and platelet count can be constructed the mode of platelet production and the platelet/megakaryocyte control system must be appreciated.

Recent studies have suggested that platelets are produced by binary sequential division of megakaryocyte cytoplasm. ${ }^{34} \mathrm{~A}$ computer simulation allows the circulating platelet volume distribution to be predicted accurately from the observed bone marrow megakaryocyte cytoplasmic volume distribution in rat, rabbit, and man. Experiments have shown that a mammal responds to a sudden loss in circulating platelet number by increasing megakaryocyte size and nuclear DNA content (ploidy). ${ }^{56}$ These larger than normal megakaryocytes produce larger than normal platelets. ${ }^{2}$ This work suggested that those patients with large platelets during acute myocardial infarction would have larger than normal megakaryocytes; we have confirmed this deduction (unpublished data). There is a highly significant correlation $(r=0.89, p<0.006)$ between circulating mean platelet volume and bone marrow mean megakaryocyte cytoplasmic volume. Moreover, subjects suffering sudden death (within a few minutes) after a coronary also had larger than normal megakaryocytes, which belong to the same statistical population as those observed after myocardial infarction.

Rate of change of circulating platelet number is determined by a balance between platelet production and destruction rate. ${ }^{7}$ When considering platelet counts and concomitant volumes it is important to distinguish between thrombokinetic equilibrium states (when the count remains constant over a substantial time) and acute dynamic responses to induced thrombocytopenic states (when the count varies with time). This aspect was not considered in the two references quoted by your correspondents. ${ }^{18}$

It can be shown that in thrombokinetic equilibrium states the platelet count, $P$, and mean platelet volume, $\mathrm{V}$, are related by the equation; $(\mathbf{P}-\mathbf{a})$ $(V-b)=c$, where $a, b$, and $c$ are constants.

This provides a rectangular hyperbolic relation between $P$ and $V$ and is a generalisation of Von Behren's relations $P V=$ constant. ${ }^{9}$ The least square error fit of platelet volume and count data shows that the experimental points lie on the asymptotes of this hyperbola, which intersect at the mean platelet volume and mean platelet count of the group considered. The characteristic indices a and b are, however, altered for different groups that are in different equilibrium states. This phenomenon arises because mean platelet volume and count have specific Gaussian distributions for a given thrombokinetic equilibrium state which combine to form a three dimensional frequency distribution of platelet count, mean volume, and frequency. The scatter diagram of platelet count and a mean volume is simply a projection of this three dimensional distribution on to two dimensional space.

In an acute response to thrombocytopenia the megakaryocytes are stimulated, increasing both their size and ploidy. The variations in megakaryocyte size and the mode of platelet production, together with the mixing of platelet populations of different mean volumes, give the observed changes in mean volume and count that have been observed in acute thrombocytopenia and thrombocytosis. ${ }^{6}$ Changes in megakaryocyte ploidy and size, reflected in changes of platelet size, are part of the normal mammalian control of haemostatic potential. Large platelets produced from stimulated megakaryocytes have an increased potential for thromboxane $A_{2}$ production per unit volume of platelet cytoplasm. ${ }^{6}$ As thromboxane $A_{2}$ is a powerful vasoconstrictor and platelet aggregator, large highly reactive platelets produced from stimulated megakaryocytes (seen after myocardial infarction and in coronary sudden death) may be causally related to coronary artery occlusion.

The importance of larger than normal platelet volume arising from stimulated megakaryocytes as a diagnostic predictor of the population at risk for myocardial infarction requires confirmation by prospective study. We do think, however, that the dismissal of the clinical relevance of the measurement of mean platelet volume has been proposed with undue haste. The knowledge gained from our recent studies suggests that such a proposal may lack scientific foundation.

JOHN MARTIN TONY TROWBRIDGE DAVID Slater

University Faculty of Medicine,
Royal Hallamshire Hospital,

Royal Hallamshire
Sheffield S10 2JF

${ }^{1}$ Levin J, Bessman JD. The inverse relation between platelet volume and platelet number. $\mathcal{F}$ Lab Clin Med $1983 ; 101: 295-307$

Martin JF, Trowbridge EA, Salmon GL, Slater DN. The relationship between platele tand megakaryocyte volumes. Thromb Res 1982;28:447-59.

Trowbridge EA, Martin JF, Slater DN, Kishk YT, biological interpretation. Thromb Res 1983;31: biological

Slater DN, Trowbridge EA, Martin JF. The megakaryocyte in thrombocytopenia: a microscopic study which supports the theory that platelets are
produced in the pulmonary circulation. Thromb Res $1983 ; 31: 163-76$.

Odell TT, Murphy JR, Jackson CW. Stimulation of megakaryocytopoiesis by acute thrombocytopenia
in rats. Blood $1976 ; 48: 765-75$. - Martin JF, Trowbridge EA, Salmon G, Plumb J.
The biological significance of platelet volume: its
relationship to bleeding time, platelet thromboxane $\mathrm{B}_{2}$ production and megakaryocyte nuclear DNA concentration. Thromb Res (in press).

Trowbridge EA, Martin JF. A biological approach to the platelet survival curve with criticism of previous

" Giles C. The platelet count and mean platelet volume. Br $\mathcal{Y}$ Haematol 1981;48:31-7. Von Behrens WE. Evidence of phytogenetic canalisa-
tion of the circulating platelet mass in man.
Thrombosis et Diathesis Haemorrhagica 1972;27: Thrombosis
159-72.

Skin test wheal size and erythema not reduced by topical antihistamine

SIR,-Dr C J Gibbs and others (12 November, p 1427) state: "It is common practice to apply a topical antihistamine preparation for symptomatic relief after skin testing with allergens." In their review of contact dermatitis, Hjorth and Fregert state: " $30 \%$ of all allergic contact dermatitis is caused or complicated by sensitivity to medicaments," including topical antibacterials, antibiotics, local anaesthetics, and various stabilisers and vehicles, as well as topical antihistamines. ${ }^{1}$ Antihistamines derived from ethylenediamine are particularly potent topical sensitisers, and once sensitisation has occurred it may sometimes recur even when the relevant drug is given by an alternative route. ${ }^{2}$

It is perhaps fortunate that atopic patients (who are likely to undergo skin tests) are no more, and perhaps less, likely to develop allergic contact dermatitis than people with normal skin, ${ }^{3}$ at least for some sensitisers (though primary irritant dermatitis is more common in atopic patients). This phenomenon may be related to $\mathrm{T}$ lymphocyte function.

The potential for skin sensitisation with topical antihistamines, together with the fact that Dr Gibbs and his colleagues found no benefit of antihistamines compared with placebo in the reduction of wheal size or symptoms, supports the statement by Wilkinson that, in relation to topical antihistamines, "there is scarcely any dermatological indication for their use.",

Clatterbridge Hospital,

NeIL H Cox Bebington,
Wirral L63 4JY

' Hiorth N, Fregert S. Contact dermatitis. In: Rook A, Wilkinson DS, Ebling FJG, eds. Textbook of dermatology. 3rd ed. Oxfor.

2 Fisher AA. Contact dermatitis. 2nd ed. Philadelphia. Lea and Febiger, 1973.

Jones HE, Lewis C, McMarlin SL. Allergic contact sensitivity in atopic dermatitis. Arch Dermatol 1973; 107:217-22.

Wilkinson DS. Topical therapy. In: Rook A, Wilkinson DS, Ebling FJG, eds. Oxford: Blackwell dermatology 3rd ed.

\section{Ventricular extrasystoles during thiazide} treatment

SIR,-The Medical Research Council working party (29 October, p 1249) states: "the explanation of the association between thiazide treatment and ventricular extrasystolic activity therefore remains uncertain" and, "certainly these results provide no conclusive evidence that either hypokalaemia itself or changes in serum potassium values have a causative role in determining ventricular extrasystoliccounts." These conclusions are surprising in that they appear to contrast with the results of the study and the presumed initial hypothesis of the investigators. The hypothesis being tested was that hypokalaemia induced by thiazides was 
associated with an increased incidence of ventricular extrasystoles. The study was not designed to establish a causal link and could not have done so. Such a link rests on a large body of evidence, referred to by the authors, establishing an electrophysiological basis for the known initiation of arrhythmias by hypokalaemia. The study showed that the serum potassium concentration was related to the ventricular extrasystolic counts when al patients were considered, that extrasystoles were commoner in those taking thiazides in the long term study, and that complex rhythm abnormalities were commoner in patients receiving bendrofluazide. Correlations were not found when decremental potassium changes were analysed. Nor were correlations found in some of the subgroups studied, but the authors themselves point out that the numbers of patients in these subgroups were small (eight to 19). A further confounding factor is that the serum potassium concentra tion was measured only once before the 24 hour tape recordings were obtained.

The authors comment that in skeletal muscle only a weak relation exists between a low serum potassium concentration and reduced intracellular potassium concentration. Cardiac muscle is different. A net potassium loss from the body caused by diuretics or diet does not change intracellular potassium concentration in the heart despite a large fall in serum potassium concentration and a loss of potassium from skeletal muscle (for references see refs 1 and 2). Diuretics in therapeutic concentrations do not have a direct effect on potassium exchange. ${ }^{1}$ If conducting tissues in the heart are similar to myocardial cells then it is the serum and not the intracellular potassium concentration that is more important in the genesis of ventricular extrasystoles in the presence of hypokalaemia.

No doubt many factors contribute to the initiation of extrasystoles, including serum potassium and magnesium concentrations, pre-existing but undiagnosed coronary artery disease, and neurogenic factors. It is impressive that in a relatively small group of patients (155 in the long term study, 228 in all) the MRC study has shown an association between ventricular extrasystoles and hypokalaemia. I do not know what the authors expected from their study and how tight the correlations were expected to be, but the negative interpretation of the results seems curious and inappropriate. The results would be equally compatible with, and provide evidence for the view that hypokalaemia induced by thiazides was an important causative factor in the initiation of ventricular extrasystoles. ${ }^{3}$

\section{Cardiothoracic Institute and National Heart Hospital \\ London W1N 2DX}

'Poole-Wilson PA, Cobbe SM, Fry CH. Acute effect of diuretics on potassium exchange, mechanical Clin $S_{c i}$ 1978;55:555-9.

${ }^{2}$ Poole-Wilson PA. The myocardial cell membrane: the effect of diuretics. $\mathcal{F} R$ Soc Med $1981 ; 44$, suppl : $9-16$.

Poole-Wilson PA. Hypokalaemia induced by thiazid diuretics in the treatment of hypertension: a cause for concern, not nihilism. Postgrad Med $\mathcal{F} 1983$
59, suppl 3:137-9.

***We sent a copy of this letter to the authors who reply below.-ED, $B M Y$.

SIR,-The main aim of the substudy was to compare the prevalence of ventricular extrasystoles in people treated with bendrofluazide with that in groups taking placebo tablets, rather than to test the hypothesis that Professor Poole-Wilson gives. The substudy did include parts which, though they could not have proved a causative relation between the concentrations of serum potassium and the ventricular extrasystolic count, could have strengthened the evidence for causality by showing, in longitudinal data, changes in these two factors occurring in the expected directions in groups of patients or in individual patients. We found no such evidence. As we said, however, and as Professor Poole-Wilson emphasises, the experimental design was such that our failure to find such evidence is far from being conclusive proof that there is no causative relation.

What impressed us about the correlations between serum potassium concentration and ventricular extrasystolic count and between serum urate concentration and ventricular extrasystolic count, was neither their strength nor their weakness but their pronounced similarity. This led us to suggest that these biochemical changes might be acting merely as markers of thiazide intake. Of course neither this similarity nor any other aspect of our results refute the hypothesis that hypokalaemia induced by thiazides is associated with an increased incidence of ventricular extrasystoles, but we would not claim that our results strengthen the evidence that there is a causative relationship between hypokalaemia and ventricular extrasystoles.

\section{MRC Working Party on Mild to Moderate Hypertension \\ Medical Unit, \\ St Mary's Hospital, \\ London $\mathrm{W}$}

W S PEART Chairman

\section{MRC Epidemiology and Medica}

Gillian GREENBERG Care Unit, Northwick Park Hospital,

Harrow HA1 3 UJ

\section{Epileptic seizures in general practice}

SIR,-We would like to reply to Dr James Edeh's letter (5 November, p 1378) concerning our paper on epilepsy in general practice (3 September, $p$ 641). In fact, our definition of active epilepsy, which was "a seizure in the previous 24 months in those with epilepsy (defined as two or more seizures)," does largely "conform to the generally accepted," and furthermore our finding of a prevalence rate for active epilepsy of 5.3/1000 is similar to those which both we and Dr Edeh quote. The separate analysis of those with single seizures, inactive epilepsy, and active epilepsy is given in our second paper.

Furthermore, we do not understand to what "controversy concerning the prevalence of epilepsy" Dr Edeh refers: the point of our article was to show that long term (usually permanent) remission occurs in most patients in whom epilepsy is diagnosed, and thus the lifetime prevalence rate (the number of patients who have ever had epilepsy) is much greater than the rate for active epilepsy, and about this there is general agreement.

Institute of Neurology

SIMON SHORVON

London

DAVID GOODRIDGE

Tonbridge,
Kent
An avoidable recurrence of cri du chat syndrome in the next generation

SIR,-There are some lessons to be learnt from the cases described by Dr J Burn and others (29 October, p 1287) and there are possible solutions.

Medical students are taught a model of the consultation that includes taking a complete history of the present illness and a complete personal, psychiatric, family, social, and geographical history followed by a full physical examination. This is usually an inefficient and ineffective method in outpatient departments and in general practice. Doctors develop either intuitively or consciously a style of solving problems in which hypotheses are created early in the consultation and are checked by further history taking, observation of non-verbal communication, and examination and investigations as necessary. Perhaps we tend to throw out the baby with the bath water.

In general practice it is appropriate that either at registration or at the first consultation with a new patient a more formal history is taken. The length of the consultation may be shortened by asking the patient to complete a health questionnaire on registration. The answers can be confirmed and augmented at the first consultation. Murray et al have shown that patients fill in the cards with reasonable accuracy and completeness. ${ }^{1}$ At the end of the first consultation the doctor can draw up a list of problems, which would, of course, include genetic problems and which should remain on the front of the patient's records for his lifetime and be available for updating. Questionnaires for patients and summary cards are available in both MRE and A4 sizes. (The central information service of the Royal College of General Practitioners would be pleased to advise.)

In our practice we are in the process of designing a family record card for every family on our list. This summarises the major problems of the family and includes a simple three generational family tree. This card is available at each consultation. An alternative solution is for patients to keep their own records or at least copies of their problem sheet.

P R V TOMSON

Abbots Langley

Murray M, Sydenham D, Westlake R. A questionnaire as a data base in problem orientated records. $\exists R$ Coll Gen Pract 1974;24:571-5.

\section{Rectal indomethacin for control of postoperative pain}

SIR,-We should like to add a word of caution to the article by Mr D J M Keenan and others (5 November, $p$ 1335) concerning the control of post-thoracotomy pain by rectal indomethacin.

Stress ulcers can occur after thoracotomy, ${ }^{1}$ and indomethacin treatment is associated with peptic ulceration. $^{2}$ The action of indomethacin, by inhibiting the production of prostaglandins, which have cytoprotective and antisecretory effects in the upper gastrointestinal tract, is mediated systemically, so no advantage in this respect is conferred by rectal administration of the drug. During the past 12 years 320 patients have been admitted to this hospital with perforated peptic ulcers. O these 55 patients were currently taking indomethacin, eight by suppository alone. When compared with 320 age-sex matched controls (eight were taking indomethacin and in only one 